\title{
A Context-Aware Model for Smart Space
}

\author{
Xianhu Tian ${ }^{1, a}$, Yong Xie ${ }^{2, b}$ \\ ${ }^{1}$ School of Huazhong University of Science and Technology, Wuhan 430074, China; \\ ${ }^{2}$ School of Huazhong University of Science and Technology, Wuhan 430074, China. \\ axianhutian@foxmail.com, byhust@163.com
}

Keywords: Smart Spaces, ontology, common context model, context reasoning.

\begin{abstract}
With mobile and pervasive computing prevails increasingly today, applications and services in smart spaces should quickly sense the context and adapt to context changing in highly dynamic environment. Personized services are also needed. Now many context models were build according to specific smart space, lacking of common structure to different fields. We propose a common ontology context model to enable the reusing and sharing of ontology. Then we propose a forward chaining reasoning method based on Rete algorithm to optimize the rule matching process. Finally we show a context model of Smart Home which was built on the common model. A rule-based reasoning test is conducted to prove the usability of the model.
\end{abstract}

\section{Introduction}

Pervasive computing [1] is a concept brought up by former chief scientist of Xerox PARC Mark Weiser, the main idea is to integrate computers seamlessly into the world and provide personized service information to users anytime, anywhere. As a typical application of pervasive computing, smart spaces have drawn researchers' attention. According to the definition given by the National Institute of Standards and Technology (NIST), smart spaces are work environments with embedded computers, information appliances, and multi-modal sensors allowing people to perform tasks efficiently by offering unprecedented levels of access to information and assistance from computers [2]. Smart spaces play an important role in pervasive computing research and provide an ideal test bed for key technologies of pervasive computing.

For the last decades, efforts have been made to research models of smart spaces which used to capturing, representing and exploiting context information. However most context models exist defect in context information sharing and context reasoning. For example: Context Toolkit [3] represents context in form of attribute-value tuples which makes context sharing hard to realize, and Cooltown [4] project proposed a context model based on web in which each object has a web description whereas the context is not extendable. To make the context information more flexible, some other models are brought up, such as CoBrA [5] and GAIA [6], but these models are designed for specific application. Since knowledge sharing and knowledge reusing between contexts [7] are vital for building a common model, ontology is used to solve the problem. Most object in smart spaces can be summarized as upper ontology or domain ontology [8, 9, 10, 11]. Wang et al [12] present an ontology based context model using OWL that can be reusing. Gu et al [13] proposed a Service-Oriented Context-Aware Middleware (SOCAM) architecture to build the ontology from top to bottom.

Although many researchers have studied context model, most of them are based on the specific application which leads to context reusing, context sharing and context reasoning problem. In this paper, we present an ontology-based context model to address critical issues including knowledge sharing, knowledge reusing and context reasoning. The context model is decoupled from the specific application scenario by layering the context ontology. Through using the rule-based context reasoning method to realize the customized service, and finally test the usability of the model by a case test. 


\section{Ontology-based context modelling of smart spaces}

The purpose of this paper is to build an ontology-based context model for smart spaces. Due to the various application of smart spaces, the ontology varies between different application filed, so the traditional ontology-based modelling can't solve the knowledge sharing and knowledge reusing problem between different domains. In this paper, context ontologies are divided into upper context ontologies and domain context ontologies. The upper context ontology is a high level ontology which captures general concept and relation in pervasive computing environment. The domain context ontology share the basic knowledge of upper context ontology, but also derives domain specific knowledge.

The upper context ontology describes five basic concepts in the smart space environment as shown in Fig.1, which are user, time, location, activity, entity. The instance of smart spaces must consist these five basic ontologies.

- User: This ontology defines the user in smart spaces and corresponding attributes such as user profile, user preference and user status.

- Time: This ontology defines the precise moment of time, duration of time or a concept of time.

- Location: This ontology defines the location of things in smart spaces or concept of places.

- Activity: This ontology defines the actions that a certain person take in smart spaces or actions that my happen in the future that can be deduced by sensed information.

- Entity: This ontology defines the things that can’t be described by User, Time, Location or Activity.

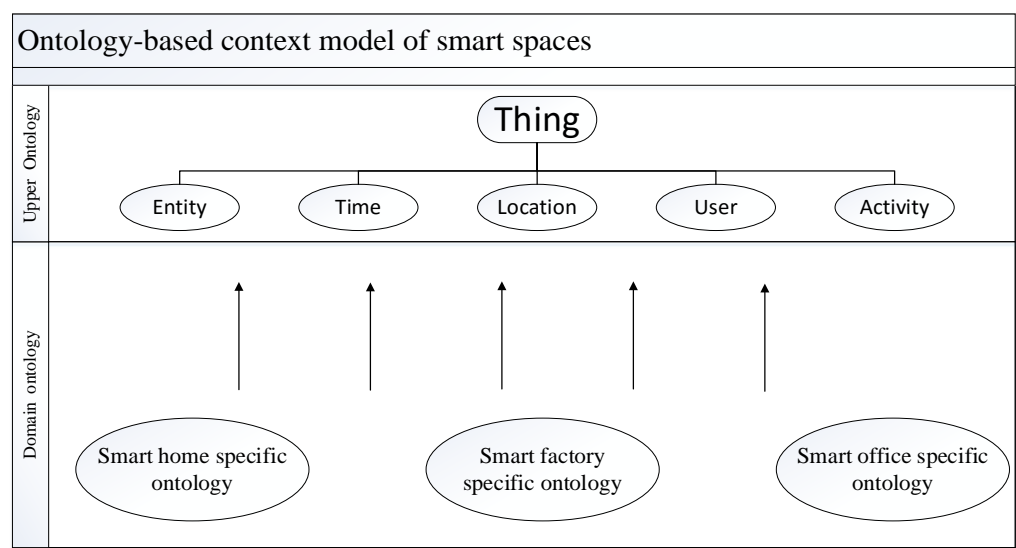

Fig.1 Smart space ontology model using two level ontology

The domain context ontology extends the upper context ontology and defines the details and additional context according to the application scenario. Since different application use the common upper context ontology, so the context reusing is achieved by hierarchy. Take the user ontology for example, user identity varies between smart home and smart factory. So extending the upper context ontology user and modify the domain ontology to reach the goal of knowledge sharing and model reusing.
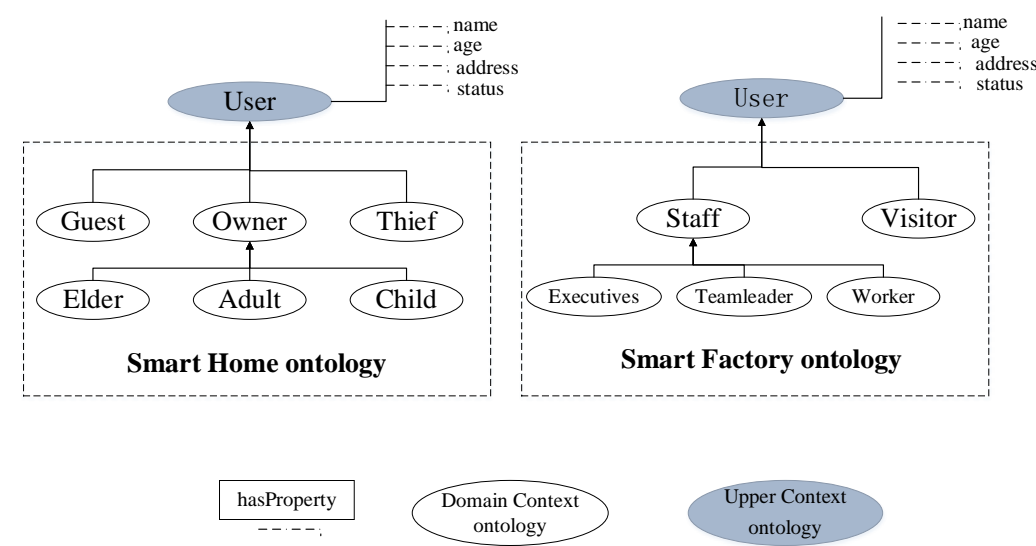

Fig.2 Domain context ontology of smart space 


\section{Rule-based context reasoning}

\subsection{Rules definition for context reasoning using SWRL}

Rule-based context reasoning broadens context information implicitly by pattern the sensed context with pre-defined rules. Rule-based context reasoning includes ontology reasoning and user-defined rule-based reasoning. The ontology reasoning is responsible for collision detection, expression optimization, ontology integration and context correctness, whereas user-defined rule-based reasoning is logical detection using rules that aim to specific application domain. User-defined rule-based reasoning ensure the flexibility and integrity of context reasoning. This paper emphasize on user-defined rule-based reasoning, selecting Semantic Web Rule Language (SWRL) proposed by W3C standard organization to support the rule-based context reasoning. SWRL rules are implies, including antecedent and consequent. Each antecedent or consequent contains zero or more context information.

It is essential to describe the context properly. In this paper, contexts are presented as first-order predicate calculus. The basic form of first-order predicate calculus is Predicate (subject, value), in which

Subject $\in S^{*}, \quad S^{*}$ : set of subject, e.g. User, Time, Activity, etc.

Predicate $\in \mathrm{V}^{*}, \mathrm{~V}^{*}$ : set of predicate name, e.g. locatedIn, hasUserStatus, etc.

Value $\in \mathrm{O}^{*}$, O*: set of all values of subject in $\mathrm{S}^{*}$, e.g. Open, Close, etc.

This paper use protégé to write SWRL, using first-order predicate calculus to describe the context, each rule can be written like this:

Antecedent -> consequent

For example: Bed(?y),User(?x), isOn(?x,?y) -> hasUserStatus(?x, rest), this rule indicates if user $\mathrm{x}$ and bed $\mathrm{y}$ exists and they has the isOn relation, then the reasoner can infer user is in rest status, namely user $x$ has attribute hasUserStatus(?x, rest).

\subsection{Rule-based context reasoning method}

The rule-based context reasoning provide forward chaining and backward chaining reasoning method. Forward chaining starts with the available data and uses user-defined rules to extract more data until a goal is reached. Backward chaining starts with a list of goals and works backwards from the consequent to the antecedent to see if there is data available that will support any of these consequents. In this paper, forward chaining reasoning is used in rule based context reasoning.

The forward chaining reasoning is based on standard Rete algorithm [14]. The idea of Rete algorithm is taking advantage of the common part in rules to reduce the storage, meanwhile memorize the temporary results to accelerate the match speed. The reasoning system using Rete algorithm is shown in Fig.3. Rete algorithm has two steps: rule compilation and rule execution. In rule compilation stage, through reading rules from the rule set to form a Rete reasoning network. In rule execution stage, passing facts to root node in Rete network, if all conditions match in a rule, the fact will reach the bottom of Rete network, indicating a rule is trigged.

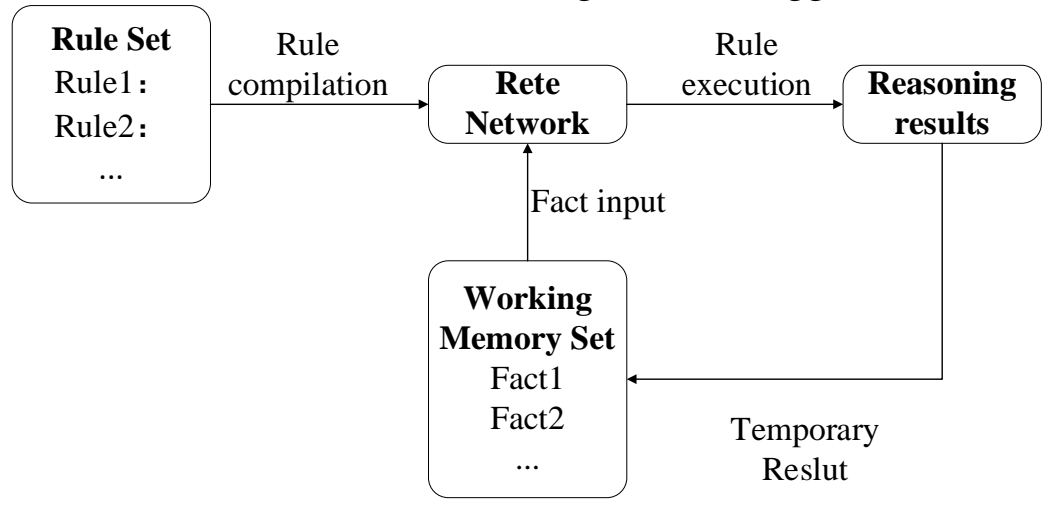

Fig.3 Reasoning System based on Rete algorithm

For example, We have the following rules:

Rule1: $(\mathrm{x})(\mathrm{y})=>$ (action1) 
Rule2: (x) (y) (z) => (action2)

A Rete network is formed based on rules above which is shown in Fig.4. The Beta node in Rete network can store the current working status of the node. When input a new fact, Beta node can match with the previous input, so the common part in rules don’t need recalculate.

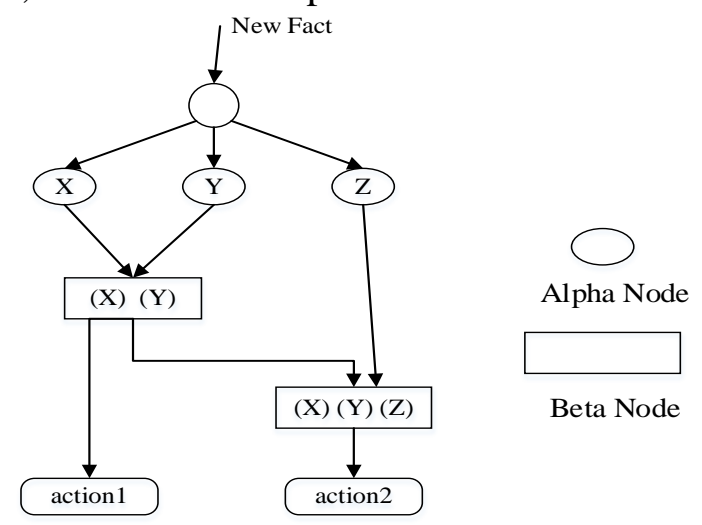

Fig.4 Rete Network Structure

\section{Case study}

Smart home is the typical application of smart spaces. In this section, we give a description of smart home scenario, then using ontology-based modelling method to describe the context in smart home. Finally, using forward chaining method based on rete algorithm to reason the context.

\subsection{Scenario description}

Smart home is built by smart object that can sense the context in pervasive computing environment and communicate with each other. When a user enters the smart home, his identity is sensed and his personal preference is loaded automatically. According to data acquired by sensors all over the room, user's status is inferred. Through user-defined rules, personized service is available such as adjust the temperature in room or adjust the light on the basis of user preference.

\subsection{Context modelling}

In the section, we use ontology and OWL to describe the context in smart home. For the purpose of better knowledge reusing and knowledge sharing, two level context architecture proposed above is applied. Since the upper context ontology is defined, we need to extends the upper context ontology and define the domain context ontology in smart home. Partial context ontology is shown in Fig.5. We use ontology-building tool protégé to build the context model and generate the context model described by OWL automatically.

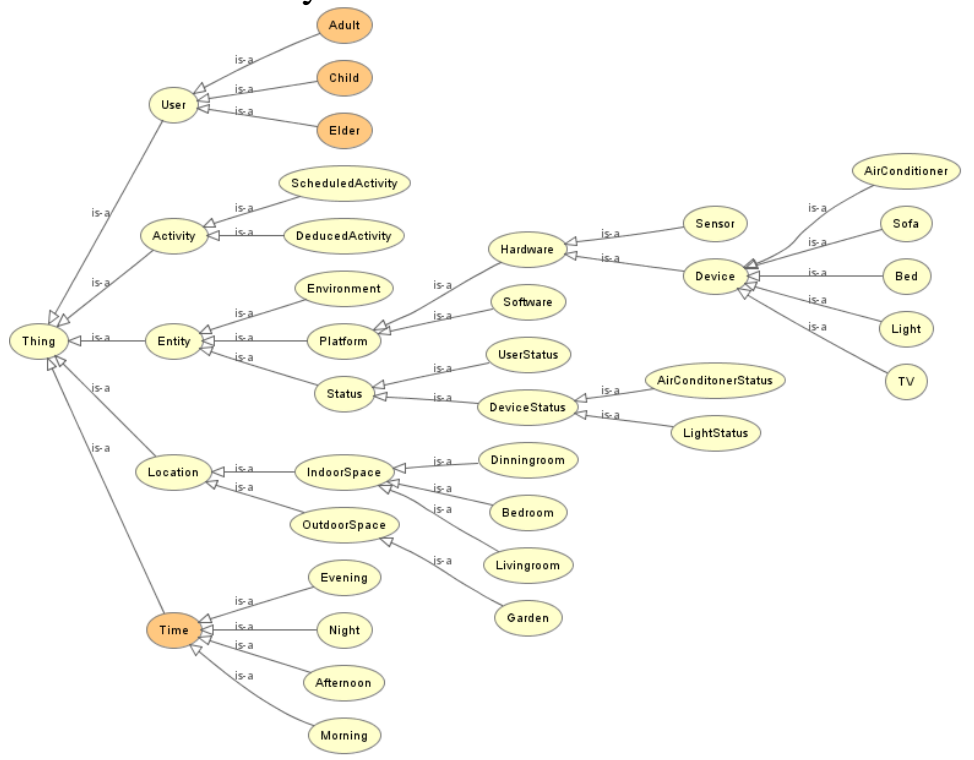

Fig.5 Partial context ontology of smart home 


\subsection{Context reasoning}

One of the advantage of our context model is the ability to support automated rule-based context reasoning. By reasoning context and add rules in our model, implicit context is deduced and personized service is realized. Because most of the rules are similar to each other, we present only one SWRL statements in this paper. Then we use a forward chaining reason engine to prove the correctness of user-defined rules.

$$
\begin{gathered}
\text { Adult(?x),AirConditioner(?y),Bedroom(?z),locatedIn(?x,?z),locatedIn(?y,?z) } \rightarrow \\
\text { hasAirConditionerStatus(?y, Cold) }
\end{gathered}
$$

In this scenario, we divide users into three categories: child, adult and elder and air conditioner in the room has two mode: mild and cold. The statement shows that if an adult enters into a room which has an air conditioner, then the air conditioner change the mode to cold.

As we mentioned above, we use protégé to build the model and we put some user-defined rules in protégé, then we use the plugged-in reason engine Pallet which support the SWRL rules to complete the reasoning process. The reasoning result is shown in Fig.6. We can see after user enter the bedroom, the air conditioner in bedroom adjust the mode into cold.

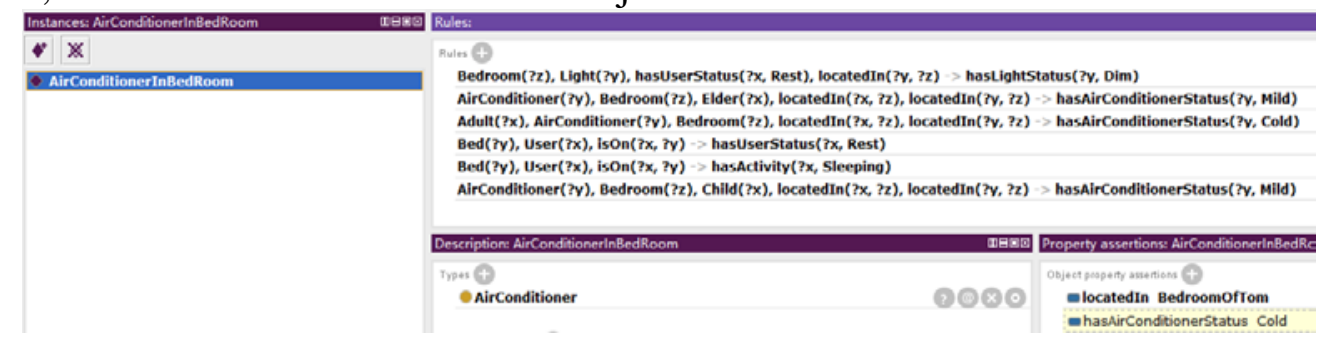

Fig.6 Air conditioner instance after reasoning

\section{Conclusion}

Context modelling is essential in smart spaces, and a number of context aware models are built in different methods. In this paper, we proposed an extensible and reusable context model with OWL using upper ontology and domain ontology to solve the knowledge sharing and knowledge reusing problem. Rules are described using SWRL to deducing the implicit context and Rete algorithm is applied to optimize the rule matching process to provide a more precise personize service. The result shows the ontology-based model is practical and reasonable.

\section{Reference}

[1] Weiser M. The computer for the 21st century[J]. Scientific american, 1991, 265(3): 94-104.

[2] NIST Smart Space Laboratory. http://www.nist.gov/smartspace/

[3] Dey A K, Abowd G D, Salber D. A conceptual framework and a toolkit for supporting the rapid prototyping of context-aware applications[J]. Human-computer interaction, 2001, 16(2): 97-166.

[4] Kindberg T, Barton J, Morgan J, et al. People, places, things: Web presence for the real world[J]. Mobile Networks and Applications, 2002, 7(5): 365-376.

[5] Chen H, Finin T, Joshi A. An ontology for context-aware pervasive computing environments[J]. The Knowledge Engineering Review, 2003, 18(03): 197-207.

[6] Ranganathan A, Campbell R H. An infrastructure for context-awareness based on first order logic[J]. Personal and Ubiquitous Computing, 2003, 7(6): 353-364.

[7] Wang X H, Zhang D Q, Gu T, et al. Ontology based context modeling and reasoning using OWL[C]//Pervasive Computing and Communications Workshops, 2004. Proceedings of the Second IEEE Annual Conference on. Ieee, 2004: 18-22.

[8] Nevatia R, Hobbs J, Bolles B. An ontology for video event representation[C]//Computer Vision 
and Pattern Recognition Workshop, 2004. CVPRW'04. Conference on. IEEE, 2004: 119-119.

[9] Yamada N, Sakamoto K, Kunito G, et al. Applying ontology and probabilistic model to human activity recognition from surrounding things[J]. Information and Media Technologies, 2007, 2(4): 1286-1297.

[10]Roy N, Gu T, Das S K. Supporting pervasive computing applications with active context fusion and semantic context delivery[J]. Pervasive and Mobile Computing, 2010, 6(1): 21-42.

[11]Gu T, Pung H K, Zhang D Q. A service - oriented middleware for building context - aware services[J]. Journal of Network and computer applications, 2005, 28(1): 1-18.

[12]Wusheng W, Weiping L, Zhonghai W, et al. An ontology-based context model for building context-aware services[C]//Intelligent Systems, Modelling and Simulation (ISMS), 2011 Second International Conference on. IEEE, 2011: 296-299.

[13]Gu T, Wang $\mathrm{X} \mathrm{H}$, Pung $\mathrm{H} \mathrm{K}$, et al. An ontology-based context model in intelligent environments[C]//Proceedings of communication networks and distributed systems modeling and simulation conference. 2004, 2004: 270-275.

[14]Forgy C L. Rete: A fast algorithm for the many pattern/many object pattern match problem[J]. Artificial intelligence, 1982, 19(1): 17-37. 